\title{
Impacto de la política fiscal en la desigualdad de género: el caso del IVA en Colombia
}

\section{Impact of Tax Policy on Gender Inequality: The case of VAT in Colombia}

\section{Impacto da política fiscal na desigualdade de gênero: o caso do IVA na Colômbia}

Diana Carolina Caicedo Enríquez 


\section{Resumen}

En este artículo se analiza cómo las políticas fiscales representan una herramienta que contribuye a la reducción o aumento de la inequidad y desigualdad de género. Para ello se abordan antecedentes sociales y normativos de atención o control a la discriminación, con especial enfoque en la esfera de lo fiscal.

Para lo anterior, se empleó un marco metodológico cualitativo con la técnica de análisis del discurso jurisprudencial, legislativo y doctrinal, permitiendo identificar las transformaciones en el ámbito internacional, que originaron los ajustes a las políticas públicas de los países; demostrando la fragilidad de las naciones en vías de desarrollo -como Colombia- ante la ineludible tarea de reforma y transformación en los ámbitos económico y tributario.

Finalmente, se evidencian los sesgos en la implementación del enfoque de género en las tres últimas reformas tributarias correspondientes a los años 2016, 2018 y 2019, al realizar un exhaustivo análisis comparativo entre estas.

Palabras clave: Tributación; Género; Desigualdad; Discriminación positiva; Principios tributarios; Sesgos; Inequidad; Política fiscal; Política pública; Justicia; Evolución; Mujer.

\section{Abstract}

This article proposes a reflection whether fiscal policy represents a tool that contributes to increase or reduce gender inequality, in order to do so, certain antecedents about social and normative facts of attention or discrimination control in general, and putting emphasis on fiscal area, are addressed.

A qualitative methodological framework with a technique of jurisprudential, legislative and doctrinal discourse was employed in order to observe changes in the international sphere, leading to adjustments and changes to public policies of many countries: fragility of developing nations, such as Colombia, can be noted to adopt modifications in economical and tax matters.

Finally, there is a reflection about bias and gender approach on the last three tax reforms corresponding to the following years: 2016, 2018 and 2019, for which a comparison is made between them.

Keywords: Taxation; Gender; Inequality; Positive Discrimination; Tax Principles; Biases; Inequity; Fiscal Policy; Public Policy; Justice; Evolution; Women. 


\section{Resumo}

Neste artigo, propõe-se refletir como as políticas fiscais representam uma ferramenta que contribui para o aumento ou redução da desigualdade de gênero, para isso, são abordados certos antecedentes de fatos sociais e normativos de atenção ou controle da discriminação em geral - e no campo fiscal - em particular.

Utilizou-se um quadro metodológico qualitativo com uma técnica de análise do discurso jurisprudencial, legislativo e doutrinário, que permite observar as transformações na esfera internacional, que levaram a ajustes nas políticas públicas dos países; além disso, a fragilidade dos países em desenvolvimento - como a Colômbia - pode ser evidenciada na adoção de mudanças nos níveis econômico e tributário.

Finalmente, refletimos sobre preconceitos e a abordagem de gênero das três últimas reformas tributárias correspondentes aos anos de 2016, 2018 e 2019, para as quais é feita uma comparação entre elas.

Palavras-chave: Tributação; Gênero; Desigualdade; Discriminação positiva; Princípios tributários; Preconceitos; Desigualdade; Política fiscal; Políticas públicas; Justiça; Evolução; Mulheres.

\section{Introducción}

La inequidad y la desigualdad en la sociedad son dos tendencias que están presentes en su accionar y evolución, además son portadoras de diferencias y problemáticas tanto entre los países como en su interior, lo cual ha sido un motivo para que los Estados, las organizaciones y las personas trabajen en políticas orientadas a atenuar su persistencia y de este modo, los efectos en las condiciones sociales. "Cuanto menos estructuradas están las desigualdades por clases sociales 'objetivas', más viva es la conciencia que de ellas se tiene y más se las vive como una amenaza subjetiva” (Dubet, 2016).

Para afrontar los efectos sociales, las Naciones Unidas, en el año 2015, fijaron unos objetivos para los países de su influencia, los cuales determinó que serán alcanzables para el año 2030, con lo que se pretende construir un mundo más equitativo e igualitario; por lo tanto se considera que se está en un momento histórico, pues los sistemas políticos y económicos predominantes compiten en ampliar su influencia en función de un mundo más justo.

Reducir la desigualdad en el mundo, tanto y dentro fuera de los países, es uno de los diecisiete objetivos que persigue la Agenda 2030 para el Desarrollo Sostenible, el programa de las Naciones Unidas que busca un futuro mejor y más sostenible para todas las personas. Sin embargo, la inequidad sigue creciendo sin parar (ONU, Noticias, 2019). 
En ese contexto, las políticas fiscales no se encuentran ajenas a las variaciones causadas por las tendencias de promoción de la igualdad y la equidad, mediante vía de la justicia y la humanización que se presentan como elementos pertinentes, donde los hechos económicos deben ser visibles; por lo tanto, los países entran en el imperativo de adaptar la legislación en esa perspectiva que sea capaz de generar cambios acordes a la coyuntura del momento y con perspectiva de largo plazo.

La política fiscal es responsable de gran parte de las diferencias entre países en materia de desigualdad. En las economías avanzadas, la política fiscal compensa alrededor de una tercera parte de la desigualdad del ingreso antes de impuestos y transferencias -lo que suele denominarse desigualdad del ingreso de mercado-, y un 75\% de ese efecto de compensación es atribuible a las transferencias. El gasto en educación y salud también incide en la desigualdad del ingreso de mercado a lo largo del tiempo, pues promueve la movilidad social, incluso entre generaciones. En las economías en desarrollo, la redistribución fiscal es mucho más deficiente debido a un gasto y a impuestos más bajos y menos progresivos (García-Escribano, 2017).

Para armonizar con los imperativos de las Naciones Unidas (ONU), en lo que respecta al componente fiscal y tributario, una característica que se determina para su aceptación y aplicación es la de tener información organizada, y que sea integral en términos de oportunidad y pertinencia para que de este modo se puedan utilizar los indicadores de las áreas económica, social y política en forma objetiva en las políticas de género, que es una de las falencias aún cierta en muchos países.

De igual manera, se debe resaltar que a la par de la discusión de la información, también la tecnología entra a dar complejidad a la dinámica de la problemática; pero lo positivo es como un número cada vez mayor de países da los pasos hacia un sistema que permita una inclusión social más eficiente; y surge el contraste de políticas públicas bastante débiles que dificultan cerrar la brecha en materia económica, por lo tanto la desigualdad sigue creciendo.

La brecha social se evidencia entonces en desigualdad salarial, de género y de oportunidades que afecta principalmente a los más vulnerables: los niños y niñas. Luchar contra esta situación no es sencillo pero tampoco imposible. Para ello, es necesario cambiar las políticas de Estado. Es imperioso que los ricos paguen impuestos y ese dinero se destine a la educación, salud y progreso de los más necesitados. Dicho cambio de mentalidad y enfoque resulta fundamental para un desarrollo sostenible (Aprendiendo Juntos, 2020).

El interés de avanzar en la comprensión y la toma de decisiones de la inequidad de género se encuentran presente y en correlación al beneficio social que requieren los países, tanto para cumplir con los Objetivos del Desarrollo Sostenible (oDs) como por la realidad de 
cada país. "La inequidad es un concepto ético e implica una valoración de la desigualdad, desde algún valor o sistema de valores” (Hernández, 2008).

Una crítica cada vez más generalizada que se realiza a los países a la hora de establecer las políticas públicas y las legislaciones en materia fiscal o tributaria, es su marcado énfasis en otorgar beneficios a las empresas, para lo cual se han diseñado planes de mejoramiento en temas tales como la facilidad de los trámites, las excepciones, los descuentos y la disminución de la carga tributaria y en cambio no se legisla para romper la inequidad, se ha evitado tocar de fondo el enfoque de género, y es cierto que cada país debe partir de su realidad.

No existe una estrategia universal. La redistribución debe reflejar las circunstancias específicas del país, como presiones fiscales subyacentes, preferencias sociales y capacidad administrativa y fiscal del gobierno. Por otra parte, los impuestos y las transferencias no pueden considerarse de manera aislada. Los países necesitan financiar las transferencias, y la combinación de instrumentos alternativos de transferencias e impuestos que escojan los países puede incidir de forma muy diferente en la equidad. Si bien algunas políticas pueden tener efectos contradictorios sobre el crecimiento y la distribución, nuestras pruebas empíricas indican que es posible lograr un crecimiento inclusivo y sostenible con una combinación adecuada de políticas. La eficiencia y la equidad deben ir de la mano (García-Escribano, 2017).

Aún en el siglo Xxi, se entiende a la igualdad de género como un tema tabú, aunque con el pasar de los años se han abierto espacios de discusión a partir de los procesos sociales y culturales; esto explica que la parte regulatoria se ha dado de manera limitada, ya que su evolución está supeditada a fuertes intereses económicos, políticos y sociales; entonces entrar a entender esa realidad con la cara de la discriminación, contribuye a dimensionar los nuevos problemas que son parte de la indignación, y esto es positivo para los gestores de políticas públicas en el camino hacia la convivencia.

Respecto a ese interés de búsqueda, hace muchos años las mujeres han desarrollado conceptos y oportunidades para que se les dignifique y se les reconozca un rol más importante dentro de la historia y la sociedad, tal como se logra evidenciar en Historia, Género y Política. Movimientos de Mujeres y Participación Política en Colombia, 1930-1991, de Lola G. Luna (1994), que cita a Teresita de Barbieri, quien “identificó la discriminación de la mujer como un problema de poder", afirmando que "En América Latina hemos privilegiado los elementos estructurales sobre los superestructurales y políticos", y de ahí se infiere que con la entrada de la mujer en el rol de tomadora de decisión a nivel político muchos temas relacionados con ellas han venido cambiando, puesto que la mujer pasó de tener un papel secundario a ser parte importante en el momento de la toma de decisiones. En la actualidad, teóricamente se busca que los ajustes en lo fiscal se dirijan hacia la aplicación integral con enfoque de género, que no induzcan a profundizar la discriminación o las desigualdades; es el caso del principio 
de equidad tributaria, principio relevante en esta época de mayor nivel de integración entre los países y comprometidos con los ods para el año 2030. Además, es un contexto de mayor conciencia social y un factor político global más fuerte sobre la necesidad de disminuir las brechas generadoras de desigualdades; pero cabe resaltar que por la falta de estructuración, información, tecnología y acuerdos internos generan problemas, ya que lo que en algunos lugares se adapta, en otros -por condiciones políticas, sociales y culturales- no se corre con la misma suerte, y por lo tanto, se tienden a dilatar los distintos procesos de gestión eficaz.

Colombia no ha sido la excepción ni se ha quedado ajena a estos procesos e inquietudes. Actualmente se ha convertido en protagonista del nuevo orden mundial; por ello, ha impulsado reformas que en teoría tienen el deber ser de coadyuvar a la cohesión social, y adaptar algunos ajustes; solo que el resultado no ha sido ejemplar, ya que han predominado los intereses económicos, capitaneados por las grandes empresas multinacionales; así lo plantean Chevallier y cita a Steranger (2014) al ilustrar sobre "el poder del mercado mundial cuando expresa que las empresas tienen necesidad del concurso de los Estados, y estos de transformarse en portavoces y defensores de los intereses económicos (competitive states). Fenómeno que se observa en el país, ya que si bien es cierto que existe la presión social de realizar cambios, estos son lentos debido a que son fuertes las firmas poderosas que pueden controlar, a través de sus hilos con el Estado y la sociedad; por lo tanto, es un logro que en el actual Plan Nacional de Desarrollo, el Gobierno Nacional haya dedicado un espacio especial para asuntos de la mujeres y también se haya exigido la transversalidad presupuestal para que todas las empresas del Estado generen programas y proyectos enfocados al empoderamiento femenino.

\section{Adaptarse al contexto frente a los desafíos mundiales}

El presente acápite tiene como finalidad exponer el estado actual de los principios tributarios, y compararlos con la situación actual de país. Por lo tanto, se partirá de la doctrina internacional, para luego analizar la legislación y jurisprudencia local.

Al analizar el caso de América Latina se encuentra que la situación de las mujeres es crítica y prueba de esto, se consta en diversos estudios:

Los índices de feminidad de la pobreza, los sesgos de género de las políticas fiscales, las brechas salariales por género y raza/etnia y la división sexual e internacional del trabajo dan cuenta de que las políticas públicas implementadas en la región siguen siendo limitadas. Por lo tanto, se requiere actuar sobre los determinantes estructurales de las brechas de desigualdad de género y evitar que, en el actual contexto de desaceleración económica y de aplicación de políticas de ajuste, se profundicen los niveles de desigualdad y se reviertan los avances obtenidos. Desigualdades de género y brechas estructurales en América Latina (Bidegain, 2016). 
En ese ámbito, se puede entender que la existencia tanto de las diferencias como los puntos en común de los países, son una realidad de su complejidad, y por lo tanto, la legislación en materia fiscal y tributaria de manera explícita o implícita, puede contener sesgos de género que acentuarían la discriminación social en contra de la mujer, por lo que resulta pertinente identificar tales sesgos en la legislación colombiana, determinar cómo es que el Estado los enfrenta y deducir conclusiones y recomendaciones.

\section{A. Cómo Colombia se está articulando en la lucha de la erradicación de la desigualdad}

La desigualdad mundial impulsó que en los últimos años las naciones afronten nuevas prioridades de atención y se centren en fenómenos y temas relacionados precisamente con combatir la desigualdad desde varios frentes; uno de ellos es el de género, que viene siendo controversial desde hace ya varios años, y en la actualidad se está haciendo énfasis en dos aspectos relevantes:

1. Economía de género.

2. Relación entre el Derecho Tributario y la desigualdad de género.

En consecuencia, se observa un sustancial crecimiento de los movimientos feministas, enfocándose en mejorar las condiciones de vida de las mujeres. Esto ha permitido realizar investigaciones de género sobre problemas, que antes habría sido imposible abordar. Un ejemplo de esto es el estudio del tema tributario y de las políticas fiscales con enfoque de género y protección de los derechos humanos.

Cuando abogamos por que haya regímenes fiscales justos y progresivos en el nivel nacional, nos damos cuenta de que las actuales leyes tributarias y las reformas propuestas se llevan a cabo dentro de existentes desigualdades de género, por lo que se discrimina a las mujeres en lo relacionado con su acceso a los ingresos, activos y bienes inmuebles y al control que tienen sobre ellos.

La política fiscal tiene que jugar un papel determinante para restablecer el equilibrio entre las desigualdades de género. Un análisis del gasto público con enfoque de género ha sido una herramienta crucial para que los defensores de la igualdad de género responsabilicen a los gobiernos (Capraro, 2015).

Estos antecedentes servirán de soporte teórico en este emprendimiento académico y social de conocer y entender cómo la política fiscal puede en su fundamentación atenuar o reforzar la discriminación y la desigualdad de género en Colombia y explorar en las medidas para afrontar los sesgos negativos, si estos llegasen a existir para que de este modo se mejore el sistema de redistribución del ingreso a través de la implementación de políticas que incluyan tratamientos diferenciales que generen una discriminación positiva. 
Existe una visión compartida entre las organizaciones de la sociedad civil y expertos/as consultados, respecto al gran reto que supone lograr políticas fiscales justas y progresivas en la región Andina y la necesidad de generar una estrategia multidimensional que transforme el discurso, los objetivos y contenidos de la política fiscal a través de la consolidación de acuerdos y alianzas entre grupos trabajando a distintos niveles de influencia política y movilización social y con diversos enfoques, pero con objetivos comunes. El proceso de consulta y diálogos que han precedido a este informe contribuye a ese objetivo al abordar el estado de los debates de la región, los enfoques en juego, las iniciativas, acciones y estrategias propuestas por las distintas partes, las alianzas, redes y actores comprometidos en esta tarea (Dejusticia, 2017).

Ahora bien, haciendo el análisis de algunos antecedentes de la sociedad colombiana, se logran apreciar los diferentes niveles de desigualdad social, como lo demuestran las cifras del DANE: en el 2018 la pobreza multidimensional fue 19,6\% para el total nacional, mientras que en el 2016 fue 17,8\%; en estos años, 1.107 .000 personas entraron a la pobreza multidimensional, con lo que se concluye que el comportamiento, según las cifras, en el país es regresivo, ya que cada año la tendencia de colombianos en la pobreza es más visible.

En la información del DANE se observa que existen diferencias marcadas entre hombres y mujeres en variables tales como: los salarios, la educación y el desempleo; con lo cual se refleja que el género femenino es uno de los grupos humanos que se encuentra bajo condiciones de "inferioridad"; además, se puede establecer que todavía las políticas públicas encaminadas a mejorar las condiciones socioeconómicas de las mujeres no son contundentes y sigue siendo uno de los puntos que menos ejecución tiene, tanto así que hay un desbalance en todo lo relacionado con el tema de género y en lo relacionado con el consumo, siendo un llamado importante para determinar cuál es el efecto que recae en este grupo social por algunas políticas económicas.

Las mujeres jóvenes desocupadas de 14 a 28 años de edad, corresponden al 47,7\% de las desocupadas; mientras que, los hombres jóvenes representan el 47,6\% de los desocupados.

Para el total nacional en el trimestre móvil agosto-octubre 2019, la diferencia entre hombres y mujeres en la tasa de desempleo para el total nacional se ubicó en -5,3 p.p. y en la tasa de ocupación en 21,5 p.p. (Departamento Administrativo Nacional de Estadistica, 2019).

En ese orden, es interesante apreciar que en Colombia, desde el comienzo de los movimientos que apalancaron el porvenir de algunos grupos sociales, entre ellos las mujeres, se ha logrado mantener y concretar la bandera por la creación de instrumentos de gestión que brinden prioridad a las políticas públicas en armonía con el Plan de Desarrollo Nacional, para de este modo participar en el desarrollo económico y social de una manera efectiva e 
integral y así, desde los distintos grupos; sobre este punto, se observa que el primer Plan Nacional de Desarrollo que incluye en un capítulo el tema de mujer, es el Plan Nacional de Desarrollo 2018-2022, Pacto por Colombia Pacto por la equidad, Ley 1955 de 2019, fundamentándose en tres Pactos Estructurales que son: la legalidad, el emprendimiento y la equidad; 13 Pactos Transversales, dentro de los cuales se encuentra el "Pacto por la equidad para las mujeres" y los 9 pactos regionales, en los que se encuentra la transversalidad del tema de la mujer.

En correspondencia con lo anterior, en el Plan Plurianual de Inversiones se destinaron 5,4 billones pesos colombianos para el Pacto por la Equidad de las Mujeres y se establecieron 8 líneas de la mujer, las cuales van desde atención a las mujeres hasta la erradicación de la violencia de género (Departamento Nacional de Planeación, 2020).

La incorporación de la perspectiva de género en la agenda pública, sumada a la búsqueda de una mayor equidad y sustentabilidad de los procesos de desarrollo, permitirán promover una mejor redistribución de los ingresos entre los géneros, la realización de los derechos sociales, una mayor participación para las mujeres y la valoración del trabajo de hombres y mujeres (Plazas, 2015).

Cabe adicionar que, desde 1991, al convertirse en un Estado Social de Derecho, Colombia ha tenido que asumir diferentes retos de inversión en los derechos humanos, sociales y económicos, lo cual ha conllevado que se establezcan los lineamientos generales, así como los mecanismos y procedimientos con los cuales procederán los planes de desarrollo y las políticas públicas; no ajeno a esto, en el ámbito de la política fiscal también se han realizado cambios que beneficien a determinados grupos sociales, en atención con los acuerdos internacionales y seguir el país hacia la vanguardia.

Precisamente en la Carta Política de 1991, que es un referente de los derechos humanos, se comienza a hablar de los Principios Tributarios en Colombia:

A partir de la Constitución Política, se establecen mandatos claros y precisos con el objeto de destinar recursos con carácter prioritario al gasto público social, cuyo objetivo es satisfacer las necesidades básicas insatisfechas, los servicios sociales básicos de educación, salud, saneamiento ambiental y agua potable, tendientes al bienestar general y al mejoramiento de la calidad de vida de la población. Toda política fiscal debe establecer claramente la relación entre la composición del gasto público y los ingresos fiscales, para el cabal cumplimiento del carácter social que se entiende tiene el Estado colombiano, relación que debe permitir financiar los gastos de funcionamiento del aparato estatal y los que se destinan a la inversión social y la promoción del desarrollo (Plazas Gómez).

Considerando que en la investigación se priorizó a nivel metodológico el enfoque cualitativo, se utilizó el análisis documental comparativo de las tres últimas reformas tributarias 
(materializadas en la Ley 1819 de 2016, Ley 1943 de 2018 y Ley 2010 de 2019), y la información del recaudo del impuesto del IVA y de cómo se compensa el pago de este impuesto a través de la creación de políticas públicas encaminadas a la inclusión social de algunos grupos, todo esto constituye un parámetro para identificar el avance que ha tenido el país en aspectos de disminución de la discriminación hacia el género femenino en materia fiscal. De igual forma, se complementó con la construcción de la matriz de correlación, en la cual se mide el impacto del impuesto a las ventas (IVA) en las personas naturales.

Teniendo en cuenta lo anterior, se encuentra que los Estándares Internacionales y la Organización para la Cooperación y el Desarrollo Económico-OCDE- han venido desarrollando requerimientos en las áreas fiscal, financiera, económica y social, los cuales podrían llegar a incidir de manera directa o indirecta en la brecha de discriminación. Es de señalar, en esta parte, que no se conoce aún si su afectación sería buena o mala, toda vez que los cambios globales se imponen y se exige que las legislaciones rompan, entre otras, las desigualdades de género, y al respecto no se han desarrollado estudios y soportes oportunos para conocer los alcances e impactos normativos; lo cual ha traído varios inconvenientes desde el momento de tener un acercamiento con la realidad, en especial cuando se hace referencia al limitante de información.

La política fiscal no es independiente del modelo económico, “expresa la estrategia de desarrollo de los gobiernos y materializa sus prioridades y la orientación de sus políticas económicas y sociales" (Oxfam, 2014,p. 5). Por ello debe fomentar la producción de riqueza y buscar la distribución y redistribución de los ingresos entre las diferentes capas de la sociedad. De cómo se obtengan los ingresos y de cómo se distribuyan, se sabrá si los ciudadanos y ciudadanas, y particularmente los grupos discriminados (como las mujeres), tienen o no un respaldo del Estado en el ejercicio de sus derechos (Plazas, 2015).

Al incorporar los antecedentes se logra ampliar la visión que Colombia ha insistido unirse a ese cambio mundial desde diversos ejes, entre los cuales se resaltan el político, el social y el económico -entre otros-, y comprender que abordar el aspecto tributario es un complemento también a resolver.

\section{B. Dónde enfatizan las reformas tributarias}

Teniendo en cuenta las apreciaciones anteriores se origina un punto de partida para entender el nuevo papel de las reformas tributarias y la erosión de la base impositiva, lo cual se constituye en el centro en torno a los ajustes tributarios, que inciden de manera directa en la vida de los ciudadanos; en consecuencia, resulta conveniente esclarecer los elementos relacionados con los tributos que inciden en la actualidad en el sensible juego de intereses y, por lo tanto, cómo determinan el peso de una reforma. 
Para ese propósito es clave partir de la siguiente pregunta: ¿De dónde surgen los inconvenientes? Básicamente, se apunta a señalar, al legislador, quien "olvidó" que antes de emitir una normatividad su deber es realizar estudios previos soportados en los hechos del país y el contexto y así no reiterar en los vacíos estructurales, que son los reclamos que se hacen desde los centros de opinión; es el caso que de manera reiterada se plasma en las reformas de los sistemas tributarios, sobre la nula o baja operatividad de los principios tributarios, se los desconoce o solamente tienen sentido teórico; por ejemplo, como ocurre con la capacidad económica y la equidad; esto es visible en la reforma tributaria de 2016 y en la reciente ley de crecimiento.

Una preocupación creciente en los últimos años es la que se genera debido a que los principios del Derecho tributario están siendo modificados y cambian obedeciendo a determinados intereses particulares, distanciándose de su razón de ser, lo que significa que estos con el paso del tiempo se van desnaturalizando; es por esto que vale recalcar que una política impositiva consistente y clara que soporte los principios es la clave para garantizar el equilibrio entre el Estado, la empresa y la sociedad.

\section{Se opacan los principios tributarios y se abre una opción}

Para Tipke (2002), experto en Derecho fiscal, los principios tienen por finalidad impartir unidad y evitar que se tomen medidas o se dicten normas contrarias a Derecho; la consecuencia de quebrantarse o violentarse es que se pueden crear privilegios o discriminaciones injustificadas; por tal razón, para encontrar una mayor equidad y óptima redistribución de los recursos, estos deben estar armonizados con la realidad y la justicia, y adaptarse a cada contexto dependiendo de la rama del Derecho de la que se esté tratando.

En este sentido, es necesario destacar el principio de igualdad, que pretende evitar "La discriminación [que] consiste en tratar a una persona, grupo o institución de manera diferente y perjudicial. Existen diferentes motivos de discriminación que a continuación desarrollaremos y todos ellos son igualmente injustos" (Tipke, 2002).

Este autor expone que históricamente en la doctrina y la práctica tributaria los tres principios de mayor relevancia han sido el de capitación, equivalencia y de capacidad económica. Para los expertos en Colombia, el sistema tributario adolece de concreción de los principios y de múltiples debilidades, como bajo nivel de recaudo, es inequitativo, es difícil de administrar y se recarga afectando el crecimiento económico.

Tal y como lo menciona Ochoa (2016), lo anterior significa que va en contravía de consolidar la justicia social y un sistema nuevo para atenuar los desfases en su aplicación, y por eso es contundente el reclamo encaminado a corregirlo y reorientarlo para encajar en el mundo estandarizado y salir del choque con los niveles de inequidad.

De igual manera, otro principio importante de resaltar es la capacidad económica, la cual no va en contravía de los derechos fundamentales, ya que coadyuva a que estos sean amparados; tal y como lo afirma Tipke (2002), este principio "No plantea qué ha hecho el Estado por el ciudadano individual, sino qué puede hacer este por el Estado". Ahora bien, 
teniendo en cuenta que el Estado colombiano es un Estado Social de Derecho, se puede afirmar que este principio toma mucha más relevancia, puesto que la finalidad inherente a nuestro tipo de Estado es la protección integral del individuo y sus derechos, por lo cual colabora en la implementación de una solución al dilema de equilibrar la relación con el mercado y la sociedad.

Por otro lado, frente al dilema de la redistribución del gasto, los principios entran a jugar un rol de armonización, ya que esta debe ser acorde con la equidad, reciprocidad e igualdad, en lo cual hay un elemento positivo, acorde con la línea que lo expresa la Corte Constitucional en su sentencia C 397 de 2011:

De acuerdo con lo previsto en el artículo 95-9 de la Constitución, con el objeto de lograr una convivencia social conforme a los valores y principios contenidos en la misma Carta Política y lograr los fines del Estado, es deber de los ciudadanos contribuir al financiamiento de los gastos e inversiones del Estado dentro de los conceptos de justicia y equidad. Este deber ciudadano tiene como fundamento "el principio de reciprocidad que rige las relaciones de los ciudadanos con el Estado y entre estos y la sociedad, a fin de equilibrar las cargas públicas que estructuran y sostienen la organización jurídico-política de la cual hacen parte, para armonizar y darle efectividad al Estado Social de Derecho".

Por su parte, con respecto al principio de equidad tributaria ha sido definido por la Corte Constitucional:

Como una manifestación específica del principio general de igualdad y comporta la proscripción de formulaciones legales que establezcan tratamientos tributarios diferenciados injustificados, ya sea porque se desconozca el mandato de igual regulación legal cuando no existan razones para un tratamiento desigual, o porque se desconozca el mandato de regulación diferenciada cuando no existan razones para un tratamiento igual. El principio de progresividad tributaria dispone que los tributos hayan de gravar de igual manera a quienes tienen la misma capacidad de pago (equidad horizontal) y han de gravar en mayor proporción a quienes disponen de una mayor capacidad contributiva (equidad vertical). En cuanto al principio de eficiencia, también ha considerado la Corte, que "resulta ser un recurso técnico del sistema tributario dirigido a lograr el mayor recaudo de tributos con un menor costo de operación; pero de otro lado, se valora como principio tributario que guía al legislador para conseguir que la imposición acarree el menor costo social para el contribuyente en el cumplimiento de su deber fiscal (gastos para llevar a cabo el pago del tributo). 
Así, el principio de progresividad tributaria en la sentencia C-385 de 2008 se define como:

La efectiva igualdad frente a las cargas tributarias se logra mediante la aplicación del principio de progresividad tributaria, en virtud del cual las leyes que establecen tributos han de gravar de igual manera a quienes tienen la misma capacidad de pago (equidad horizontal) y han de gravar en mayor proporción a quienes disponen de una mayor capacidad contributiva (equidad vertical), que propende por que el sistema sea justo, en forma tal que el sacrificio que corresponda a unos y otros sea equivalente, si se tiene en cuenta la capacidad económica de cada contribuyente.

En el sentido del respecto de los principios tributarios, se logra observar que se construye una crítica interesante de mostrar en el caso colombiano y es la que establece:

Las últimas reformas tributarias señalan una intención de sustituir los principios tributarios establecidos en la Constitución Política por otros formulados o recomendados por organismos internacionales como el Fondo Monetario Internacional (FMI) y la Organización para la Cooperación y el Desarrollo Económico (OCDE). Un ejemplo claro de ello es la reforma tributaria 1819 de 2016, la cual busca llevar los tributos bajo principios como simplicidad y competitividad (Moreno, 2017).

En ese orden de ideas, se observa en los párrafos citados anteriormente de las sentencias, cómo la jurisprudencia de la Corte Constitucional ha venido refiriéndose a los principios que deben regir el sistema tributario, y menciona: legalidad, certeza e irretroactividad, además de equidad, eficiencia y progresividad; de ello se deduce que los principios determinan la legitimidad del sistema en su conjunto y no de un impuesto en particular. Este es un hecho de visión social, de apertura teórica y un aporte doctrinal, hacia una postura activa de la política fiscal, encaminada a incidir en las condiciones sociales de justicia y equidad que requiere el país.

\section{La igualdad como camino para políticas fiscales más incluyentes}

En este apartado se busca sustentar que la implementación del principio de igualdad en las políticas fiscales conduce a sistemas tributarios más incluyentes. Para lo anterior, primero se partirá de la perspectiva de género a nivel internacional y después, se aterrizará la implementación en Colombia.

\section{A. El soporte de la igualdad en la reivindicación femenina}

En este punto se pretende dar el alcance de eje central al concepto de igualdad sobre el que se sustentan las reivindicaciones de los movimientos feministas en búsqueda de un 
tratamiento equitativo entre los géneros, especialmente en las políticas públicas y su énfasis de estos movimientos en el propósito por el interés en la política fiscal.

A lo largo del texto se relacionan algunos avances significativos en la acción social reivindicatoria, materializados en declaraciones de las Naciones Unidas y en sus agendas de objetivos comunes. También, se presentan algunas consideraciones sobre los progresos de la equidad de género en América Latina y en especial en Colombia.

Por último, se muestra cómo los asuntos fiscales constituyen un aspecto fundamental en las políticas públicas para promover la equidad, volviéndose esta clase de políticas cada vez más sensibles en la agenda pública desde la perspectiva de género.

\section{B. La búsqueda de mayor igualdad como objetivo de la comunidad internacional}

La lucha por la equidad se remonta a siglos atrás, cuando hombres y mujeres comenzaron a reclamar una sociedad más equitativa y justa, logrando establecer la igualdad como un derecho que se potenció hasta alcanzar la dimensión global, hace más de sesenta años, con la promulgación de la Declaración Universal de los Derechos Humanos de la onu del año de 1948.

Es de tener claro que aplicar ese objetivo constituye un esfuerzo, que se asume desde los ingresos fiscales; por lo tanto, los impuestos son necesarios para garantizar los derechos y no solo para fines recaudatorios. El Estado debe atender, como en este caso, la desigualdad y discriminación, imponiendo el "sacrificio" a un grupo en favor de otros (Sustein, 2011). Este argumento estaría consagrando la necesidad de hacer esfuerzos de discriminación positiva a favor de la mujer y desde esa perspectiva asumir un rol en la sociedad con autonomía, derechos y obligaciones.

Adicional a lo anterior, la igualdad está vinculada muy cerca a otros principios, tales como la justicia, la equidad y la libertad. Un logro importante que va encaminado en esta dirección ha sido la inclusión de metas de igualdad y equidad en los Objetivos del Milenio, que posteriormente se convirtieron en los actuales Objetivos de Desarrollo Sostenible.

Sin embargo, en la historia de la humanidad han sido reiterados los atropellos contra el derecho a la igualdad y particularmente a la equidad entre los géneros, por lo que no es accidental que tanto en los Objetivos del Milenio como en los de Desarrollo Sostenible se intente corregir esa problemática, posición que se refleja en objetivos específicos:

En los Objetivos del Milenio:

Objetivo 3: Promover la igualdad entre los sexos y la autonomía de la mujer.

Y en los Objetivos de Desarrollo Sostenible encontramos que la iniciativa se amplía y se encuentran ya no un solo objetivo sino dos, los cuales son:

Objetivo 5: Igualdad de género.

Objetivo 10: Reducción de las desigualdades.

Por consiguiente, en los últimos años, el tema de igualdad de género es uno de los incluidos en las agendas y en el foro político de alto nivel de la onU, donde el oDs 5 fue 
priorizado en 2017, mas no en el año 2019; sin embargo, dentro de las 12 metas urgentes para la movilización de recursos se encuentra la meta 5.c. que busca aprobar y fortalecer políticas para promover la igualdad de género y el empoderamiento de todas las mujeres y las niñas en todos los niveles.

En consecuencia, los países han incluido en sus agendas acciones más concretas. Al respecto, en Colombia se consagran en los planes de desarrollo nacional, departamentales y municipales; son explícitas las metas que hacen alusión a las mujeres, se especifican las que están en estado de embarazo; nutrición; mortalidad materna; acceso universal a servicios de salud sexual y reproductiva; embarazo adolescente; acceso igualitario de hombres y mujeres a la formación técnica, profesional y superior de calidad; hombres y mujeres alfabetizados; conocimientos teóricos y prácticos entre otros, sobre igualdad de género; acceso a métodos de saneamiento adecuados; empleo pleno, productivo y decente; igualdad de remuneración a trabajo igual; derechos laborales de mujeres migrantes; espacios públicos seguros para mujeres y niños, e información estadística desagregada por género.

\section{Desigualdad de género en América Latina}

En los últimos años, el mundo y en especial América Latina se enfrentan a un lento crecimiento económico, lo cual repercute en crecientes desigualdades sociales.

Los panoramas social y económico no son excluyentes y se vuelven vulnerables cuando la desigualdad es persistente independiente de su cuantía, y es por ello que la búsqueda de su control o erradicación se impone para las nuevas generaciones de la gran América Latina.

Durante la última década, en algunos países de la región, la reducción de la desigualdad de ingresos y de los índices de pobreza se produjo simultáneamente con el aumento de la feminidad de la pobreza. El índice de feminidad muestra las disparidades en la incidencia de la pobreza en mujeres y varones (de 20 a 59 años), medida en términos de ingreso. Es posible afirmar que las políticas tendientes a reducirla no han impactado de la misma forma en hombres y en mujeres. Desde 2012, la tendencia a la reducción de la pobreza se ha estancado en América Latina, mientras que el índice de feminidad sigue aumentando. Es decir que tanto en periodos de reducción de la pobreza como en periodos de estancamiento, la pobreza sigue afectando en mayor grado a las mujeres que a los hombres, en edades críticas en términos productivos y reproductivos. En 2014, por cada 100 hombres que vivían en hogares pobres en la región, había 118 mujeres en la misma situación (Bidegain, 2016).

Entonces, es visible la problemática planteada con conexiones en distintas manifestaciones sociales, culturales, políticas, y económicas, campos estos que apuntan hacia la indignación general e indica que nos enfrentamos a un cambio de época: continuar con las mismas prácticas políticas y propósitos de concebir el mundo, que por los hechos ya no es viable, o comprometerse por su transformación hacia renovados paradigmas de desarrollo 
hasta llegar a un verdadero desarrollo sostenible con equidad social, inclusión y visión de largo plazo.

En América Latina, los índices de feminidad de la pobreza, los sesgos de género de las políticas fiscales, las brechas salariales por género y raza/etnia y la división sexual e internacional del trabajo dan cuenta de que las políticas públicas implementadas en la región siguen siendo limitadas. Por lo tanto, se requiere actuar sobre los determinantes estructurales de las brechas de desigualdad de género y evitar que, en el actual contexto de desaceleración económica y de aplicación de políticas de ajuste, se profundicen los niveles de desigualdad y se reviertan los avances obtenidos (Bidegain, 2016).

Teniendo en cuenta lo anterior, aunque América Latina no es la región más pobre del mundo, sí es la más desigual (CEPAL, 2018) y, por lo tanto, se debe atender porque es una limitante para el progreso y avance de la sociedad.

El índice de feminidad en hogares pobres compara el porcentaje de mujeres pobres de 20 a 59 años respecto de los hombres pobres en esa misma franja y en sus resultados se muestra que los esfuerzos de reducción de la pobreza en la región no han beneficiado de igual manera a hombres y mujeres, ni ha tenido el mismo ritmo, y que finalmente los hogares pobres concentran una mayor proporción de mujeres en edades de mayor demanda productiva y reproductiva.

En 2017, por cada 100 hombres viviendo en hogares pobres en la región, había 113 mujeres en similar situación, esto evidencia la falta de autonomía económica de las mujeres, quienes en ausencia de otros ingresos del hogar son más proclives a estar en situación de pobreza, situación que se agudiza en hogares con mayor presencia de niños y niñas.

La desigualdad es fuente de secuelas que pueden ser estructurales o de coyuntura; es el caso del efecto del cambio climático, la inflación y la desigual redistribución del ingreso, entre otros hechos, que recaen con dureza en los eslabones más pobres de la sociedad y en especial en las mujeres, dado que históricamente son las más afectadas por la brecha de desigualdad en la región.

\section{Luchas y avances de las mujeres en Colombia}

Algunos antecedentes de la sociedad colombiana permiten apreciar diferentes niveles de desigualdad social: se observa que algunos sectores han sido excluidos y de manera constante la mujer, que está ocupando los espacios más adversos, sus condiciones generales muy lentamente cambian y, además, se presentan indicadores que limitan sus medios de consumo por efecto de algunas políticas, una de las cuales podría ser la tributaria. 
No obstante lo expuesto, Colombia ha insistido en la atención de la situación: acoge convenios internacionales, el principal de los cuales es la Convención sobre la Eliminación de toda forma de Discriminación contra la Mujer (CEDAw, siglas en inglés), que se ratificó en 1982 y su protocolo en el año 2007; los convenios de la Organización Internacional del Trabajo (OIT) vinculados con la igualdad de género se han ratificado tres: el C100, que habla sobre la igualdad de remuneración; el C111, sobre la discriminación, y el C189, de trabajadoras y trabajadores domésticos; no han sido ratificados el C115, sobre los trabajadores con responsabilidades familiares; el C183, que trata sobre la protección de la maternidad, y el C190, sobre la eliminación de la violencia y el acoso en el mundo del trabajo (onu Mujeres, 2019).

En esa dirección, se espera que este trabajo entre en el ejercicio de complementar la visión y entender la discriminación de género; lo que permite que se explore el fenómeno desde los aportes internacionales, los principios tributarios, el marco de la reforma tributaria y desde el modelo matemático, de tal manera que se pueda identificar el efecto del IVA en las personas naturales en sede de la perspectiva de género.

Ahora bien, al hablar de género también se debe comprender como posibilidad de encuentro, de reconocimiento, regulación de las relaciones humanas, garantía para ejercer el derecho a la palabra, oportunidad de justicia en términos de ofrecer o recibir una justificación, y como solidaridad; de igual manera, puede considerarse como una oportunidad valiosa para la transformación de la sociedad, por su contribución a múltiples transiciones de violencias y desigualdades destructivas a convenios sociales por el cambio y la inclusión.

En Colombia, ese proceso de reconocimiento se ha encaminado por la convergencia de acciones populares y gubernamentales. Así, con relación a los impuestos, fue el resultado de la presión de grupos de ciudadanos como el de "menstruación libre de impuestos", que en noviembre de 2018 la Corte Constitucional, en la Sentencia C-117declaró la inconstitucionalidad del Iva del 5\% de los tampones y toallas higiénicas; se consideró un hecho histórico y el país entró a ser protagonista del nuevo orden mundial en las reivindicaciones de género.

En la mencionada sentencia, que constituye un avance importante en la materia y que tiene relación intrínseca con la presente investigación, el órgano fallador establece:

El principio de igualdad gobierna las diferentes relaciones entre el Estado y los individuos, así como entre los mismos sujetos. Su contenido, como es bien sabido, es de carácter relacional e involucra: (i) el deber de prodigar tratamiento análogo a los sujetos que están en condiciones relevantes similares; (ii) la procedencia del tratamiento jurídico diverso a los mismos sujetos o situaciones, cuando sus condiciones fácticas son disímiles, y (iii) la obligación de asegurar la eficacia de los derechos de aquellas personas o grupos tradicionalmente discriminados, o que están en situación de debilidad manifiesta. 
En el devenir de las reivindicaciones y en la actualidad parece que ante la opinión pública nacional e internacional, Colombia ofrece un momento favorable respecto al diálogo constructivo, donde las mujeres están teniendo más espacios. Al progresar la mujer podrá generar soluciones a los conflictos en territorios y sociedades complejas, ya que al ser incluida en la creación de políticas públicas será un referente de valor para el Estado Social de Derecho que se consolida.

Otro paso en esa dirección es a raíz del Plan Nacional de Desarrollo del periodo 20182022, que en el artículo 155 se incluye el enfoque diferencial en el Sistema Estadístico Nacional y se crean los trazadores presupuestales para las asignaciones presupuestales de los pueblos étnicos y de equidad de género, se fortalece la institucionalidad de género para las mujeres en Colombia, y es el puente operativo con el sistema de planeación, desde donde se puede hacer el monitoreo público.

A partir del año 2019 se aplicó en Colombia Atenea (onu Mujeres, 2019), que son mecanismos para acelerar la participación política paritaria de las mujeres en América Latina, es una iniciativa que busca incentivar a las mujeres en el ejercicio del derecho de participación política en condiciones de igualdad y no discriminación; para su ejecución, Colombia ha suscrito los principales instrumentos que garantizan los derechos políticos de las mujeres, este hecho se fortaleció, porque el país en 2019 por primera vez tiene un gabinete ministerial paritario.

\section{E. La política tributaria con perspectiva de género}

En el contexto de reafirmación de los derechos fundamentales, en particular, con relación a la equidad y la igualdad, han sido visibles los movimientos que buscan revindicar los derechos de las distintas comunidades, incluidos los movimientos feministas, enfocados a mejorar las condiciones de vida de las mujeres en la sociedad. Una de las expresiones de estos movimientos ha sido la lucha para que las políticas públicas incluyan la perspectiva de género.

Es oportuno anotar que una de las políticas más relevantes de los gobiernos por su impacto sobre la equidad e igualdad es la política fiscal, y en ese terreno las demandas del movimiento feminista han alcanzado inicialmente algunos desarrollos teóricos y resultados prácticos en lo relativo a la discusión, diseño y aplicación del gasto público.

Los estudios de género en el otro componente fiscal de los ingresos están menos desarrollados, situación que justifica promover investigaciones de género en materia tributaria, a fin de examinar si los impuestos en Colombia, por razones meramente sexuales, otorgan privilegios o se asignan cargas mayores a uno u otro género.

Cuando abogamos por que haya regímenes fiscales justos y progresivos en el nivel nacional, nos damos cuenta de que las actuales leyes tributarias y las reformas propuestas se llevan a cabo dentro de existentes desigualdades de género, por lo que se 
discrimina a las mujeres en lo relacionado con su acceso a los ingresos, activos y bienes inmuebles y al control que tienen sobre ellos.

La política fiscal tiene que jugar un papel determinante para restablecer el equilibrio entre las desigualdades de género. Un análisis del gasto público con enfoque de género ha sido una herramienta crucial para que los defensores de la igualdad de género responsabilicen a los gobiernos (Capraro, 2015).

En el caso de la desigualdad causada por la tributación comenzó a generar un interés particular hace casi tres décadas en el mundo -es relativamente reciente y más en Colombia-, ya que fue desde 1993, al abrigo de los movimientos feministas que surgió en la comunidad académica una preocupación por los posibles sesgos de género contenidos en las políticas tributarias. Los trabajos desarrollados por Janet Stotsky (1993, 1995 y 2005) plantearon la posibilidad de que la tributación contuviera sesgos positivos o negativos en favor de hombres y mujeres.

Los reclamos feministas y los oportunos estudios son alicientes para explorar las evidencias en la normatividad acerca de la existencia de sesgos implícitos, que, sin aludir directamente a uno de los géneros, terminan por impactar positiva o negativamente la situación de hombres o mujeres.

\section{El enfoque de género en las reformas tributarias de 2016 y 2018, la correlación del IVA y las personas naturales}

\section{A. Las reformas Ley 1819 de 2016 y leyes 1943 de 2018 y 2010 de 2019}

Las reformas tributarias han tendido a consolidar los impuestos indirectos como el factor relevante de los ingresos del Estado, tanto por la facilidad del recaudo como la simplicidad; ello, aunque es obvio que los impuestos indirectos son regresivos, no contempla los principios tributarios, en especial uno de los principios fundamentales, como es la progresividad (Moreno, 2017), de manera que ese carácter puede llevar a discriminar a determinados sectores sociales.

Teniendo en cuenta esa premisa, también su utilización y alcance se ha transmitido a Colombia, dado que en los últimos años la carga impositiva tiende a recaer en gran medida en las personas naturales, y el IVA entró a convertirse en un soporte del fisco y de la tendencia que se mencionó. Esto, en las condiciones socio-económicas del país, es la mujer fácilmente afectada, y no se descarta que se enfrente a una situación compleja,

Problemática que se encuentra relacionada con los desequilibrios macroeconómicos que son consecuencia de los desajustes fiscales, que buscan ser solucionados con el incremento de la carga impositiva a través de los impuestos indirectos, el aumento de las servicios públicos y la reducción de gastos a través de la aplicación de la Regla Fiscal. Es evidente que la desigualdad e inequidad ha generado un trato desfavorable y 
discriminatorio para las mujeres. Sumado a lo anterior, se evidencia que la calidad de vida de las mujeres no ha mejorado en Colombia, pues a pesar de que se ha reducido la pobreza, que pasó de tener una cifra del 48\% en el año 2008 al 29,3\% en el 2014 (DANE, 2014), la pobreza y la desigualdad sigue teniendo rostro de mujer, tal y como registra en los datos estadísticos del DANE y la CEPAL, según los cuales, las mujeres entre 20 a 59 años de edad exceden en un $30 \%$ o más la tasa de pobreza, en comparación con la de los hombres de edad similar (CEPAL, 2014). Según la CEPAL, una de cada tres mujeres no tiene ingresos propios, en comparación con solo el 11,7\% de los hombres; y 118 mujeres por cada 100 hombres viven en situación de pobreza (CEPAL, 2014; Plazas, 2015).

Las consideraciones mencionadas se pueden explorar en las tres últimas reformas tributarias (consignadas en la Ley 1819 de 2016, Ley 1943 de 2018 y Ley 2010 de2019), dado que son el eje para materializar su alcance y representan las bases de legalidad y afianzan el enfoque del sistema tributario que los gobiernos impulsan, acorde con los lineamientos de sostenibilidad fiscal y los fines económicos y sociales.

En las tablas 1 y 2 se ilustra una demarcación de las leyes tributarias: Ley 1819 de 2016, Ley 1943 de 2018 y Ley 2010 de 2019:

- En la tabla 1, se presentan la descripción, los principios y el tipo de sesgo implícito o explícito, así como la calificación de fuerte, débil y ausente.

- En la tabla 2 se observa el sesgo en el IVA, se aprecia un avance en 2016 y vuelve a retroceder en 2018, es decir, se necesita continuar en el debate para tratar estos temas en las políticas tributarias.

Tabla 1. EnfoQue de género y UbiCaCión del SESGo 2020

\begin{tabular}{|c|c|c|c|c|}
\hline Ley tributaria & Descripción & Principios & Sesgo implícito & Sesgo explícito \\
\hline Ley 1819 de 2016. & $\begin{array}{l}\text { Se fortalecen los me- } \\
\text { canismos para la lucha } \\
\text { contra la evasión y la } \\
\text { elusión fiscal, a partir } \\
\text { de una reforma tribu- } \\
\text { taria estructural. }\end{array}$ & $\begin{array}{l}\text { Legalidad: Fuerte. } \\
\text { Equidad: Débil. } \\
\text { Capacidad Económi- } \\
\text { ca: Débil. } \\
\text { Igualdad: Débil. } \\
\text { Progresividad: Débil. } \\
\text { Eficiencia: Fuerte. } \\
\text { Justicia: Ausente. }\end{array}$ & $\begin{array}{l}\text { Presente en los artícu- } \\
\text { los que contienen la va- } \\
\text { riación de la tarifa del } \\
\text { IVA con los cuales se } \\
\text { infiere que se afecta a } \\
\text { las personas de más es- } \\
\text { casos recursos, además } \\
\text { cambió las exenciones } \\
\text { en materia de renta, en- } \\
\text { tre otros aspectos. }\end{array}$ & $\begin{array}{l}\text { Se incluyen las com- } \\
\text { presas y tampones de } \\
\text { uso femenino en el IVA } \\
\text { del } 5 \% \text {. } \\
\text { Hay medidas que be- } \\
\text { nefician a la mujer, ya } \\
\text { que hay exenciones en } \\
\text { las ESAL para quienes } \\
\text { trabajen con este gru- } \\
\text { po poblacional. }\end{array}$ \\
\hline Ley 1943 de 2018. & $\begin{array}{l}\text { Establece normas de } \\
\text { financiamiento para } \\
\text { el restablecimiento } \\
\text { del equilibrio del pre- } \\
\text { supuesto general. }\end{array}$ & $\begin{array}{l}\text { Legalidad: Fuerte. } \\
\text { Equidad: Débil. } \\
\text { Capacidad Económi- } \\
\text { ca: Débil. } \\
\text { Igualdad: Débil. } \\
\text { Progresividad: Débil. } \\
\text { Eficiencia: Fuerte. } \\
\text { Justicia: Ausente. }\end{array}$ & $\begin{array}{l}\text { Hay variaciones en la } \\
\text { tarifa de renta, entre } \\
\text { otras medidas. }\end{array}$ & No hay. \\
\hline
\end{tabular}




\begin{tabular}{|c|c|c|c|c|}
\hline Ley tributaria & Descripción & Principios & Sesgo implícito & Sesgo explícito \\
\hline Ley 2010 de 2019. & $\begin{array}{l}\text { Determina normas } \\
\text { para la promoción } \\
\text { del crecimiento eco- } \\
\text { nómico, el empleo, la } \\
\text { inversión, el fortaleci- } \\
\text { miento de las finanzas } \\
\text { públicas y la progre- } \\
\text { sividad, equidad y } \\
\text { eficiencia del sistema } \\
\text { tributario, de acuerdo } \\
\text { con los objetivos que } \\
\text { sobre la materia im- } \\
\text { pulsaron la Ley } 1943 \\
\text { de } 2018 \text {. }\end{array}$ & $\begin{array}{l}\text { Legalidad: Fuerte. } \\
\text { Equidad: Débil. } \\
\text { Capacidad económi- } \\
\text { ca: Débil. } \\
\text { Igualdad: Débil. } \\
\text { Progresividad: Débil. } \\
\text { Eficiencia: Fuerte. } \\
\text { Justicia: Ausente. }\end{array}$ & $\begin{array}{l}\text { Hay variaciones de las } \\
\text { tarifas de renta, las ba- } \\
\text { ses para retención en } \\
\text { la fuente; sin embar- } \\
\text { go, se tiene un punto } \\
\text { positivo: la reducción } \\
\text { gradual de aportes a } \\
\text { pensión. }\end{array}$ & No hay. \\
\hline
\end{tabular}

Elaboración: Fuente propia a partir de las normas tributarias.

Tabla 2. Ubicación SESGo IVA

\begin{tabular}{|l|l|l|}
\hline \multicolumn{1}{|c|}{ Ley tributaria } & \multicolumn{1}{c|}{ Sesgo } & \multicolumn{1}{c|}{ Artículo } \\
\hline Ley 1819 de 2016. & $\begin{array}{l}\text { Explícito. } \\
\text { Explícito positivo. } \\
\text { Implícito. }\end{array}$ & $\begin{array}{l}185 . \\
175 \text { y } 152 . \\
184 \text { y otros. }\end{array}$ \\
\hline Ley 1943 de 2018. & Implícito. & 22,24 y 26, etc. \\
\hline Ley 2010 de 2019. & Implícito. & $27,28,29,30,31$ y 32. \\
\hline
\end{tabular}

Fuente: Elaboración propia a partir de las normas tributarias.

Por su parte, en el trabajo de campo que se realizó en un supermercado de la ciudad de Pasto, que representa una ciudad de ingresos medios de Colombia, se observa que los precios tienen una tendencia superior en los bienes elaborados para el consumo de mujeres, que aquellos de consumo de los hombres. Esto no es una casualidad, pues en 2015 el estudio de Clara Plazas ilustra para Bogotá; de esta situación surgen preguntas que inducen a avanzar en investigaciones complementarias, que es la herramienta apta para dilucidar cómo hacer políticas públicas incluyentes, y, además, entender en qué nivel impacta la cultura en la persistente discriminación de género.

\section{Conclusiones}

De la investigación se deduce, en primer lugar, la tendencia a la discriminación de género en el sistema tributario, y se advierte la amplificación del fenómeno a diversos campos de la sociedad; de ello, dan cuenta diversos estudios en los ámbitos nacional e internacional. 
En segundo término, se advierte una actividad notable de los movimientos feministas y de organismos internacionales orientados a contrarrestar la inequidad y desigualdad de género y la búsqueda de alternativas para enfrentar ese fenómeno.

En tercera instancia, Colombia no ha sido ajena a las tendencias internacionales en las que se reivindican los derechos de las mujeres y, en tal sentido, se deben mencionar las políticas de género impulsadas por el sector público, en atención a la acción de los acuerdos internacionales y los movimientos feministas, entre las que sobresalen las políticas presupuestarias, programas con visión de género, y el monitoreo a través del Departamento Nacional de Planeación (DNP), el Departamento Nacional de Estadísticas (DANE) y el Ministerio de Hacienda. Esos esfuerzos se pueden identificar de manera más detallada en los párrafos siguientes.

La igualdad es un derecho fundamental, que se ha venido fortaleciendo a lo largo de los años en la mayoría de los países del mundo occidental.

Los Estados, las organizaciones internacionales y las organizaciones civiles permanentemente están estudiando los avances y las problemáticas existentes en torno a la aplicación del derecho a la igualdad y el principio de no discriminación, gracias a lo cual encuentran argumentos para crear, eliminar o modificar mecanismos propensos a la discriminación y desigualdad, de acuerdo con los artículos 1 y 2 de la Declaración Universal de los Derechos Humanos, que estipula que las naciones deberán defender a los ciudadanos ante violaciones a la igualdad.

Paralelamente, los ciudadanos, mediante las luchas sociales, han buscado poner en la agenda pública los tipos de discriminación económico-social o implícita en la ley, que afectan la equidad e igualdad. Por consiguiente, los movimientos sociales pretenden enfrentar los diferentes temas en los cuales se observa una desventaja de algunos grupos, como el de las mujeres, en el cual se resaltan problemas en relación con la equidad vertical y horizontal tributaria y se logra concluir que los beneficios no son uniformes.

En relación con la materia tributaria, se identifica que las mujeres no cuentan con políticas tributarias justas, y se resalta que esto ocasiona que ellas no logren alcanzar la satisfacción de las necesidades, y por ello, se genera distorsión e inequidad en el sistema tributario; por lo cual se busca generar voluntad política para que desde los Estados y los organismos internacionales fomenten realizar las correcciones necesarias.

Sobre el control ante la inequidad y la desigualdad, además se direcciona modernizar la administración tributaria para optimizar, facilitar y consolidar la fiscalización, en armonía con modificaciones al régimen tributario para que se adopten las medidas de atención a la desigualdad social.

En el caso colombiano han existido interesantes avances, desde el Congreso de la República, y en particular la Comisión Legal para la Equidad de la Mujer, en su compromiso con la garantía de los derechos de las mujeres y la construcción de igualdad entre mujeres y hombres, su trabajo en los últimos años.

La Convención sobre la Eliminación de todas las Formas de Discriminación contra la Mujer (CEDAw), aprobada en Colombia mediante la Ley 51 de 1981, es el tratado 
internacional vinculante más amplio sobre derechos de las mujeres y la igualdad de género. Su objetivo es lograr la igualdad de jure y de facto entre hombres y mujeres en el goce de sus derechos humanos y las libertades fundamentales, es decir, tanto en las normas y las leyes, como en los hechos.

Es de resaltar que el movimiento y la lucha feminista llegó a los estrados judiciales en la Sentencia C-117/18, lo cual influyó para la inexequibilidad de la partida 96.19 del artículo 185 de la Ley 1819 de 2016, que grava las toallas higiénicas y tampones con la tarifa del 5\% del IVA e incluir estos productos en el listado de bienes exentos del impuesto del valor agregado.

Como un soporte al monitoreo de los compromisos, se solicita al DNP un informe sobre los recursos de inversión en el proyecto del PGN 2020 destinados al avance país con relación a las 9 metas del Objetivo de Desarrollo Sostenible (oDs) 5. Lograr la igualdad de género y empoderar a todas las mujeres y las niñas, y metas de otros oDs, como la meta 3,7 y 8.5 , identificando aportes de entidades líderes y entidades acompañantes, en los términos establecidos en el CONPES 3918 de 2018.

La academia debe involucrarse en el análisis de género a las Rentas de Destinación Específica, el costo fiscal de los Gastos Tributarios, de cara a la posibilidad de poner sobre el debate público la financiación de la política pública de mujeres y equidad de género, más allá de la estrategia de transversalización.

En Colombia, el Plan Nacional de Desarrollo 2018-2019, "Pacto por Colombia, Pacto por la Equidad", presenta el pacto XIV sobre la Equidad de las Mujeres, al que se asignan en el Plan Nacional de Inversiones Públicas 2019- 2022 recursos por un monto de \$5.400 miles de millones, y se hace la aclaración de que esos valores no suman al total por ser recursos transversales en los diferentes pactos.

En este sentido, el artículo 221 de la ley anteriormente citada, Trazador presupuestal para la equidad de la mujer, establece que las entidades que integran el PGN ubiquen en este trazador las asignaciones presupuestales a estos efectos, para que el Ministerio de Hacienda y Crédito Público y el Departamento Nacional de Planeación consoliden y presenten al Congreso de la República un informe con dicha información, proyectos que también serán identificados en el Plan Operativo Anual de Inversiones respectivo. Y en el artículo siguiente, 222, crea el Sistema nacional de mujeres que agrupa las diferentes ramas del poder público en torno a políticas públicas a favor de la igualdad entre mujeres y hombres. En este se le asigna al Sistema la función de hacer seguimiento a la política pública de cuidado.

En el Pacto XIV, acerca de la equidad de las mujeres del actual Plan Nacional de Desarrollo, tiene un carácter transversal, con una asignación de recursos de la misma naturaleza, para el periodo 208-2022, de \$5.400 mil millones. En el documento "Bases del Plan Nacional de Desarrollo 2018 - 2022: Pacto por Colombia, pacto por la equidad", anexo de la Ley 1955 de 2019, se presentan 8 líneas de política.

El Gasto Público Social evidencia que el Estado lo aplica para neutralizar la desigualdad y discriminación de género; es una práctica que impulsan los organismos internacionales y 
las presiones de los movimientos feministas, que muestran auge en sus reivindicaciones; así, el Estado lo asume como el instrumento idóneo, limitando el papel del sistema tributario en su carácter de política pública como potenciador de contrarrestar la discriminación de género y contribuir a la equidad e igualdad.

De las últimas reformas tributarias de 2016, Ley 1819, y las reformas de 2018 y 2019 , leyes 1943 y 2010, respectivamente, se deduce que en la reforma de 2016 se introduce un enfoque de género limitado, explícito para algunos Bienes de la mujer y constituye un sesgo positivo, es un avance normativo que se refuerza con la Sentencia C-117 de 2019, de la Corte Constitucional.

Las leyes de 2018 y 2019 tienen una orientación clásica, la Ley 1943 de 2018 fue declarada inconstitucional y sus lineamientos básicos se consignaron en la nueva Ley 2010 de 2019: en común enfatizan en el recaudo, la lucha contra la evasión y elusión y el énfasis del crecimiento económico, dan prelación a principios de simplicidad y competitividad y se alejan del principio de capacidad económica, se generan sesgos negativos y se da un retroceso en relación con el enfoque de género, que trató la Ley 1819 de2016. Estas reformas han sido objeto de críticas y han motivado reacciones de rechazo por los movimientos sociales.

Como un elemento complementario, el fenómeno de los precios brinda pautas para ampliar el estudio de la inequidad de género; se observa que la discriminación es compleja por la multiplicidad de causas, los precios de los mismos bienes son diferentes para mujeres y hombres. Las preguntas pueden ser diversas: ¿será cultural y cuál es el mensaje para construir políticas públicas incluyentes?

Finalmente, se evidencia que Colombia es un país que necesita realismo, investigación y participación para solucionar sus problemas, presenta grandes desigualdades, y la desigualdad económica afecta más a la mujer, como grupo social vulnerable. En consecuencia, para salir de esa situación es imperativo buscar instrumentos de participación, democráticos, y tocar la política fiscal para alcanzar resultados sostenibles; en este sentido, se impone reducir el efecto socio-económico adverso con medidas de discriminación positiva, fomentar nuevas formas de consumo, basadas en el minimalismo. Es entrar en la línea de encausar las políticas por una cultura ética de producción y consumo, que contribuya a la convivencia e inclusión.

\section{Referencias}

Aprendiendo Juntos (febrero 05 de 2020). Educo. Recuperado el 07 de febrero de 2020, de https://www.educo.org/Blog/Categoria/Aprendiendo-juntos

Arenas, A. (2018). La política tributaria y los sesgos de género: aproximaciones al caso colombiano. Bogotá D.C: Fescol. 
Benavente, M. (2014). Políticas públicas para la igualdad de género: un aporte a la autonomía de las mujeres. Santiago de Chile: Naciones Unidas, CEPAL \& Cooperación Española.

Bidegain, N. (2016). Desigualdades de género y brechas estructurales en América Latina. Nueva Sociedad.

Capraro, C. (2015). Impuestos a hombres y mujeres: por qué el enfoque de género es crucial para un régimen fiscal justo. Christian Aid.

CEPAL (2018). La Agenda 2030 y los Objetivos de Desarrollo Sostenible Una oportunidad para América Latina y el Caribe. Santiago de Chile: CEPAL.

Chevallier, J. (2014). El Estado Posmoderno. Bogotá D.C: Universidad Externado de Colombia.

Dejusticia, L. O. (2017). Informe de una serie de diálogos organizados.

Departamento Administrativo Nacional de Estadística (2019). Información laboral Agosto-Octubre 2019. Bogotá D.C: DANE.

Departamento Nacional de Planeación (febrero de 2020). Plan Nacional de Desarrollo: Pactos Transversales. Recuperado el 7 de febrero de 2020, de https://www.dnp. gov.co/DNPN/Plan-Nacional-de-Desarrollo/Paginas/Pactos-Transversales/Pacto-de-equidad-para-las-mujeres/Equidad-para-mujeres.aspx

Dubet, F. (2016). ¿Por qué preferimos la desigualdad? (aunque digamos lo contrario). Buenos Aires: Siglo Veintuno.

García-Escribano, V. G. (11 de Octubre de 2017). Desigualdad: la política fiscal puede corregir la situación. Recuperado el 7 de febrero de 2020, de Diálogo a Fondo: https://blog-dialogoafondo.imf.org/?p=8430

Hernández, M. (2008). Desigualdad, inequidad e injusticia en el debate actual en Ciudad de México: Salud Capital. Ciudad de México.

Luna, Lola G. (1994). Historia Género y Política. Movimientos de mujeres y participación Política en Colombia 1930-1991. Barcelona: Universidad de Barcelona.

Moreno, A. (2017). Principios Tributarios establecidos en el ordenamiento jurídico vs el Impuesto sobre la Renta. Bogotá D.C: Universidad Libre. 
ONU Noticias (8 de febrero de 2019). ONU NOTICIAS. Recuperado el 7 de febrero de 2020, de https://news.un.org/es/story/2019/02/1450741

ONu Mujeres (2019). Atenea por una democracia 50/50 . Colombia la hora de paridad. Bogotá D.C: onu Mujeres.

Plazas, C. (2015). Política fiscal y género: que no se descargue la crisis sobre las mujeres. Bogotá D.C.: Universidad del Rosario.

Sustein, S. (2011). El Costo de los Derechos: por qué la libertad depende de los impuestos. Buenos Aires: Siglo Veintiuno.

Tipke, K. (2002). Moral tributaria del Estado y de los contribuyentes. Madrid: Marcial Pons. 